\title{
Successful Treatment of Paraplegia in Spinal Tuberculosis: Case Study and Literature Review
}

\section{Sager SHY Hanna* Sameh Ibrahim Al Shinawy, Islam Elsayed Aly and Aliaa Fareed Khaja}

Department Orthopedic Surgery, Al Razi Orthopedic Hospital, Kuwait Institute for Medical Specializations, Kuwait

*Corresponding Author: Sager SHY Hanna, Department Orthopedic Surgery, Al Razi Orthopedic Hospital, Kuwait Institute for Medical Specializations, Kuwait.
Received: March 16, 2020

Published: March 20, 2020

(C) All rights are reserved by Sager SHY

Hanna., et al.

\begin{abstract}
Although uncommon, extrapulmonary tuberculosis is not a rare occurrence. Bone and joint involvement constitute at least ten percent of cases, with the most affected site being the spine. Patients with spinal tuberculosis usually present with back pain, with other pulmonary or constitutional symptoms being absent, resulting in a difficult diagnosis. This report highlights the challenges experienced in the diagnosis of spinal tuberculosis. We report the case of a 28-year old male paraplegic who presented with deteriorating symptoms after being bedridden for a year as he was a drug addict. The patient suffered from a fixed neck flexion complete deformity. Imaging studies showed a large soft tissue mass in front of C2 up to C7 with destruction of C2 and C2/3 subluxation, multiple vertebral osteolytic lesions mostly at T2/3 with massive T2 destruction and large epidural collection compressing the cord. Surgery with posterior decompression at level of T1-T2 with fixation from C1 to T5. In the same setting an anterior evacuation of the cervicalabcess ws done, huge amounts of pus was evacuated. Post-operatively, a right pneumothorax was diagnosed and the patient was placed in the Intensive Care Unit. The patient had 3/5 lower limb muscle power after two weeks. Biopsy and cultures confirmed Tuberculosis spondylodiscitis. Considering the complications associated with untreated spinal tuberculosis with paraplegia and the importance of surgery, it is pertinent to state that high suspicion plays a critical role in early diagnosis of such cases.
\end{abstract}

Keywords: Paraplegia; Tuberculosis

\section{Background}

Tuberculosis is a major cause of infectious ailments that affects at least a third of the global population [1]. Even in developed nations like the United States, no less than 9,412 cases of tuberculosis were recorded in 2014. Although, there has been a decrease in the incidence of tuberculosis, the number of cases can be said to be the fewest in a decade. In the United States, geographical regions with the highest rates of tuberculosis include Florida, New York, Texas, and California [2].

The occurrence of TB in extrapulmonary sites is uncommon but not rare. These may be triggered by contact with infected fluids (or secretions) through the respiratory and gastrointestinal tract, hematogenous and/or lymphogenous dissemination, and contiguous spread [3]. The involvement of the skeletal system accounts for up to ten percent of extrapulmonary tuberculosis cases. Of these, the spine is most affected. Spinal tuberculosis is more prevalent in endemic nations, while in developed countries it occurs more in older adults. Overall, spinal tuberculosis is not as common in the Western World, but upon presenting, it is most prevalent in the immigrant population as well as the HIV population. Spinal tuberculosis is characterized by a wide range of symptoms; the most frequent being back pain. Other symptoms may include weight loss, malaise, night sweats, and neurologic symptoms with paraplegia being the 
most severe. Diagnosis is confirmed by imaging or the presence of bacilli (acid-fast) on culture or microscopy [4]. The challenge encountered in the diagnosis of tuberculosis is that it does not receive consideration in the event of a vague clinical presentation. This article reports the case of a 28-year old tuberculosis spine patient who found relief via surgical intervention.

\section{Case Presentation}

A 28-year old male presented to Al Razi Orthopedic casualty with deteriorating symptoms after having been quadraplegic and bedridden for a year with urine and bowel incontinence. His motor function was 3/5 (upper limb) and 2/5 (lower limb). The patient was known to be a drug abuser and had refused medical care before that, thus his condition deteriorated to the state of being bedridden.

The patient had noticed a swelling several years ago that he described as being a soft, painless mass, not exceeding $1 \mathrm{~cm}$ in size in the anterior aspect of his neck. There was no report of redness, warmth, or fluid/pus discharge. A year before presentation at our facility, the patient briefly experienced cough and fever, and was relieved after standard supportive, but the cough persisted for two months. For the next several weeks, he experienced pain around the ribs, which radiated to the back. The back pain could be described as "writhing." Pain killers were administered, and he was advised to rest. The pain persisted even after rest and worsened after a night's sleep or a short nap. The patient found it difficult to get up after sleep and this hurt both his gait and walking pace. In the evenings, the patient experienced low-grade fevers and a gradual loss of weight from 54 kilograms to 44 kilograms.

\section{Investigations}

As part of the initial investigations, a complete blood count with white cell differentials, Erythrocyte sedimentation rate(ESR, urea and electrolytes, and a coagulation profile were obtained. In addition, a Mantoux Test as well as a Tubercular IgA test, and ASO titers were ordered. Blood and urine cultures were also ordered. Results showed anemia, leucopenia, and a moderate rise in ESR (52 $\mathrm{mm})$. Plain radiographs of the chest, cervical, lumbar and dorsal spine were also added. The Anterior-Posterior (Figure 1) and lateral radiographs of the cervical spine (Figure 2) showed loss of lordotic curvature of the spine as well as destruction of the vertebral bodies and right deviation of the trachea. Further imaging was therefore requested; a Computed Tomography and Magnetic Resonance Imaging of the entire spine.

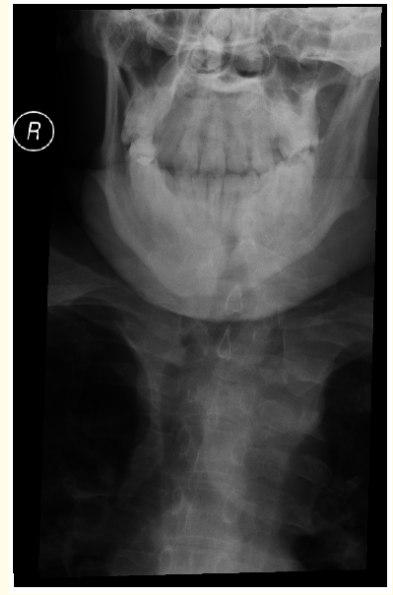

Figure 1: AP Cervical Spine radiograph

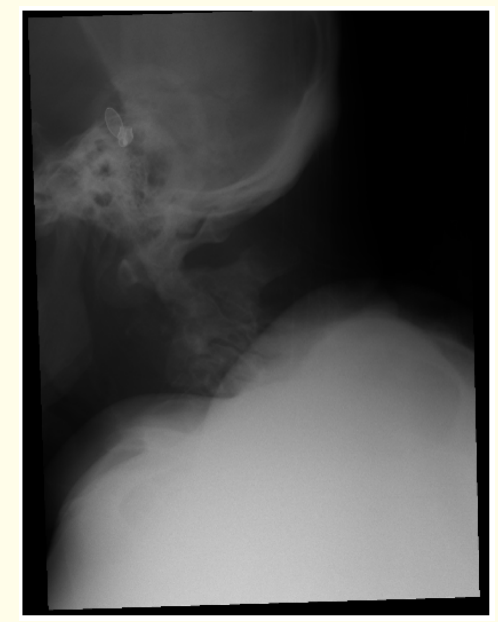

Figure 2: Lateral Cervical Spine Radiograph.

CT scan of the cervical spine revealed anterior subluxation of third cervical vertebra with destruction of body of C2 and upper endplate of C3 (Figure 3). In addition, the loss of curvature of the cervical spine was further delineated and kyphotic segment was seen at cervical 7- thoracic 1 vertebra due to destruction of the adjacent vertebral bodies. Large anterior soft tissue masses were seen indenting the trachea and causing right sided deviation. The CT of the thoraco-lumbar spine revealed multiple sites of vertebral body lytic destruction of the sixth thoracic vertebra as well as the fifth lumbar vertebra. MRI cervical spine with contrast showed the 
extent and size of these soft tissue collections found to be anterior vertebral as well as epidural abscesses, spinal cord compression at c2-3, c7-t1 and the myelomalacia associated with such compression. The thoracic and lumbar spine MRI showed high signal intensity in the 6ixth thoracic vertebra and fifth lumbar spine with no associated abscess formation (Figure 4).

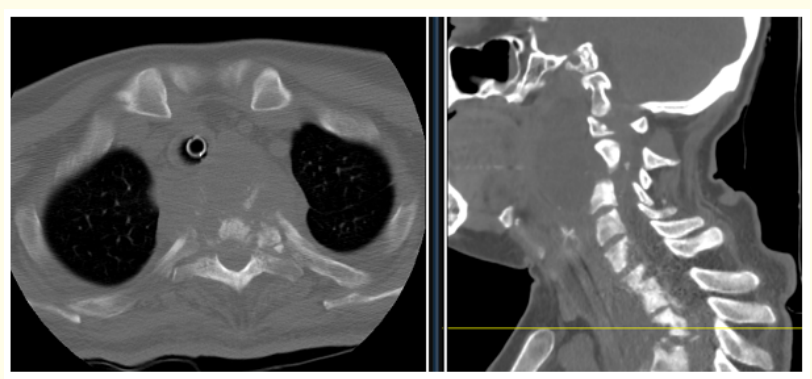

Figure 3: Cervical spine Mid-sagittal CT scan.

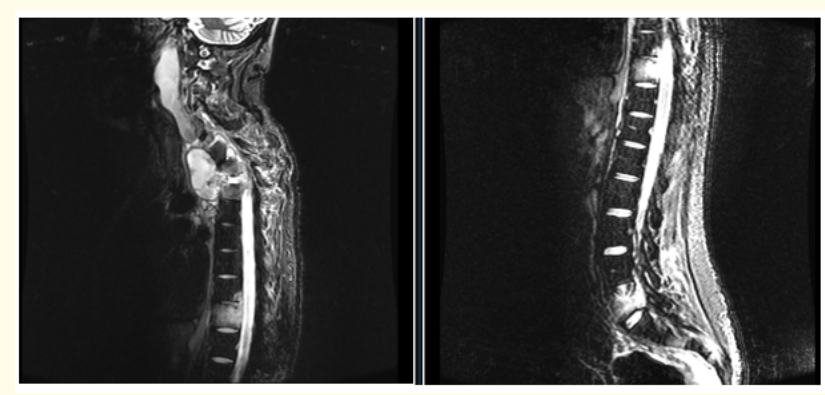

Figure 4: Whole Spine MRI with contrast STIR.

\section{Differential diagnosis}

At this point, the differential diagnosis included both bacterial spondylitis and TB spondylitis. Due to the long history of the disease and associated with iliopsoas inflammation seen in the MRI, TB was considered first in differential diagnosis. CT-guided aspiration by the interventional radiologists was done and cultures proved positive for M. tuberculosis.

\section{Treatment}

Treatment for tis patient required a multi-disciplinary team approach involving spine surgeons, infectious diseases physicians, and interventional radiologists. Standard anti-tubercular treatment was initiated after positive cultures.
A trial of drainage of his abscess under ultrasound guidance was done but only a small amount of turbid, thick fluid was collected. Thus, the patient was taken to surgery for posterior decompression and long segment fixation from the first cervical vertebra to the fifth thoracic vertebra. During the same stage, the patient was turned for a Smith Robinson approach for an anterior decompression of the large abscess at the level of the junction of the second and third cervical vertebra. Posterior cervical fixation was done at $\mathrm{C} 1$ by lateral mass screws, $\mathrm{C} 2$ by pedicle screws, lateral mass screws from C3 to C6, and pedicle screws from C7 to T5. In addition, samples from bone, soft tissue and abscess was sent for culture and sensitivity. Patient was taken to the Intensive care Unit post-operatively. His stay in the ICU was long and included right-sided recurrent pneumothoraces and right lung collapse (Figure 5).
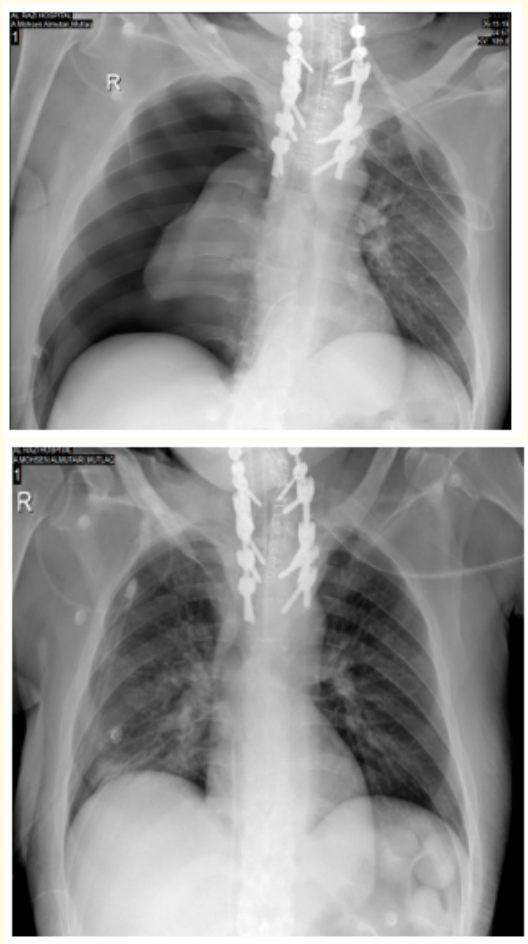

Figure 5: Right-sided pneumothorax, treated with chest drain.

\section{Follow-up}

Two weeks after returning to the ward, the patient's movement function returned back to 545 for both upper and lower limbs, and sensation was $3 / 5$ for upper limb, while lower limb sensation was $2 / 5$. 
At three month follow up, sensation in his lower limb improved to $3 / 5$. At six month follow up, the motor function returned to $5 / 5$. At 14 months follow up, the sensation returned to $5 / 5$, and he was back to independent urine and bowel function.

\section{Discussion}

Spinal tuberculosis is not common in the Western world, which explains why it would be overlooked by medical professionals. However, there are a few risk factors that should raise suspicion for tuberculosis. Factors that predispose an individual to TB also raise their risk for spinal tuberculosis. These include social and economic factors like overcrowding, poverty, illiteracy, malnutrition, diabetes mellitus, alcoholism, HIV, and other clinical conditions that suppress the immune system [4].

Spinal TB patients also have non-specific symptoms that cause a potential delay in diagnosis $[7,8]$. The patient's medical history is of great importance but not completely dependable for making a prompt diagnosis. The most common presentation of spinal tuberculosis back pain $[4,5]$. Back pain has an insidious onset while exhibiting some kind of mechanical nature during the start of the disease. Local tenderness, persistent spinal pain, limited mobility of the spine, neurologic complications such as paralysis, and a febrile state present with the progression of destruction. The progression of these symptoms occurs gradually, with the average duration of illness ranging from $5-12$ months. Most cases do not present with constitutional symptoms but may include weight loss, general malaise, fatigue, and night sweats. Neurologic symptoms include numbness and weakness, which can result in total paraplegia if not treated on time. The incidence of neurological sequelae as a consequence of spinal TB varies greatly, from as low as 10 percent to as high as 76 percent [6]. Radiologically, the anterior inferior vertebral body is first affected, followed by the central, anterior, sparing the vertebral discs, with the lower thoracic and the upper lumbar spine being the most common sites affected [4].

In most cases, the clinical presentation, along with the spine's radiologic appearance and a positive result from a tuberculin test, may indicate spinal tuberculosis, but confirmation of the diagnosis is evident by the presence of acid-fast bacilli from the body fluids or the bone [11]. There is a need for a high degree of suspicion to prevent delays that may lead to neurological complications, per- manent damage, and a high rate of mortality. Delays and misdiagnoses are common.

Several studies report that surgery is the major treatment and is recommended for complications including loss of sensation and quadraplegia (as in our case), due to abscesses, spinal tuberculoma, or spinal tuberculosis. Late diagnosis is associated with poor prognosis and an increased need for surgical intervention. Although advanced MRI technology should improve diagnosis time, there's been a consistent delay in the diagnosis of spinal tuberculosis. Also, MRI does not help with the differentiation between malignancy and infection $[12,13]$. Similar results after surgical debridement, decompression and fixation in the spine have been reported with complete recovery $[14,15]$.

Early disease may be treated with a conservative approach, but the prevention of neurological consequences requires surgical intervention. Neurosurgical intervention corrects existent deformities, enables spinal cord decompression, addresses any destabilization of the spine, and allows abscess debridement [12]. Recently, spinal tuberculosis has been grouped into three main types (Type IA/IB. II, and III). This classification is based on 7 radiological and clinical features. The least severe is Type IA with the disease restricted to the vertebrae. Type III is more extensive, characterized by the collapse of the vertebrae, formation of an abscess, and deformities. Surgical intervention is recommended for types IB, II and III. When there is a spinal cold abscess, conservative therapy is not adequate for the prevention of vertebral destruction. There is a need for surgical drainage. Epidural abscesses are risk factors for neurological defects and require immediate drainage to prevent compression of the cord. The recent system of classification is important and if applied in the initial stages of the disease, can help determine whether surgery is needed for the prevention of complex neurological complications such as paraplegia [16].

\section{Conclusion}

In advanced nations, back pain is a common disorder, and also the most common symptom of spinal tuberculosis. It is important to note that TB is a curable disease and neurology is reversible and less virulent than bacterial spondylitis which has less rewarding results. Careful consideration of TB diagnosis and assessment of risk factors can avoid diagnostic delays and mismanagement. This can decrease the neurological complications and the need for extensive surgical interventions. 


\section{Bibliography}

1. CDC-TB-Data and Statistics, Cdc.gov (2016).

2. Scott C., et al. "Tuberculosis trends-United States, 2014". Morbidity and Mortality Weekly Report 64.10 (2015): 265-269.

3. Bennett J., et al. "Douglas, and Bennett's Principles and Practice of Infectious Diseases". London, UK: Elsevier Health Sciences (2014).

4. Garg RK and Somvanshi DS. "Spinal tuberculosis: a review". The Journal of Spinal Cord Medicine 34.5 (2011): 440-454.

5. Ferrer MF., et al. "Tuberculosis of the spine. A systematic review of case series". International Orthopaedics 36.2 (2012): 221-231.

6. Rasouli MR., et al. "Spinal tuberculosis: diagnosis and management”. Asian Spine Journal 6.4 (2012): 294-308.

7. ES Nussbaum., et al. "Spinal tuberculosis: a diagnostic and management challenge". Journal of Neurosurgery 83 (1995): 243-247.

8. MS Moon. "Tuberculosis of the spine. Controversies and a new challenge". Spine 22.15 (1997): 1791-1797.

9. SM Tuli., et al. "Tuberculosis of the spine". Acta Orthopaedica Scandinavica 38 (1967): 445-458.

10. L Huelskamp., et al. "TB of the spine: Pott's disease”. Orthopaedic Nursing 19 (2000): 31-35.

11. JP Janssens and R de Haller. "Spinal tuberculosis in a developed country. A review of 26 cases with special emphasis on abscess and neurologic complications". Clinical Orthopaedics and Related Research 257 (1990): 67-75.

12. Ekinci S., et al. "Spinal Tuberculosis". Journal of Experimental Neuroscience (2015): 89-90.

13. Moon MS. "Tuberculosis of spine: current views in diagnosis and management". Asian Spine Journal 8.1 (2014): 97-111.

14. Jain A., et al. "Tuberculosis of spine (C1 to D4)". Spinal Cord 37.5 (1999): 362-369.

15. Kirshblum SC and O'Connor K.C. "Predicting neurologic recovery in traumatic cervical spinal cord injury". Archives of Physical Medicine and Rehabilitation 79.11 (1998): 1456-1466.
16. Oguz E., et al. "A new classification and guide for surgical treatment of spinal tuberculosis". International Orthopaedics 32.1 (2008): 127-133.

\section{Assets from publication with us}

- Prompt Acknowledgement after receiving the article

- Thorough Double blinded peer review

- Rapid Publication

- Issue of Publication Certificate

- High visibility of your Published work

Website: www.actascientific.com/

Submit Article: www.actascientific.com/submission.php

Email us: editor@actascientific.com

Contact us: +919182824667 